\title{
SEARCHES FOR BINARY STARS IN GLOBULAR CLUSTERS
}

\author{
C. PRYOR
}

Rutgers, the State University of New Jersey

Dept. of Physics \& Astronomy, P.O. Box 849, Piscataway, NJ 08855-0849 USA

J.M. FLETCHER, J.E. HESSER, R.D MCCLURE, P.B. STETSON Dominion Astrophysical Observatory, $H I A / N R C$

H.B. RICHER, G.G. FAHLMAN, R.A. IBATA, N.C. IVANANS, AND G. MANDUSHEV

Univ. of British Columbia, Dept. of Geophysics \& Astronomy

R.A. BELL

Univ. of Maryland, Dept. of Astronomy

M. BOLTE

Univ. of California, Lick Observatory

H.E. BOND

Space Telescope Science Institute

W.E. HARRIS

McMaster Univ., Dept. of Physics \& Astronomy

D.A. VANDENBERG

Univ. of Victoria, Dept. of Physics \& Astronomy

AND

M.A. WOOD

Florida Institute of Technology, Dept. of Physics \& Space Science

\section{Introduction}

Primordial binaries in globular clusters are important both because their properties are an integral part of the description of the stellar population and because they can strongly influence the dynamical evolution of the cluster (see Hut, this volume). 
Binary stars have been identified in globular clusters using $\mathrm{x}$-ray emission, eclipses, radial velocity variations, and their position in the colormagnitude diagram (cmd). The last few years have seen rapid progress in identifying eclipsing variables using large sets of CCD images. The deep cmds of the centers of clusters produced from Hubble Space Telescope (HST) images, many of them shown at this conference, are poised to revolutionize the identification of "binary sequences" in the cmd. Surveys for radial velocity variables are being extended to fainter stars and longer time baselines. Each search technique has its strengths and weaknesses. Surveys for eclipsing systems are currently a relatively unique window into the number of short-period binaries, binary sequences are sensitive to systems with a wide range of periods, and radial velocity surveys can, eventually, determine the distributions of orbital properties. The interesting task ahead for the next few years will be integrating these disparate data to build a more complete picture of the binary star populations in globular clusters.

What we want to know is the frequency of binaries. How does their number vary from cluster to cluster and how does it vary with position within a cluster? In conjunction with this, we also want to know the distribution of binaries in the "internal" properties of primary mass, mass ratio, period, and eccentricity. These are still relatively distant goals. This paper briefly reviews what we do know and the prospects for further progress.

\section{Radial Velocity Variables and the Binary Frequency}

Radial velocity surveys of luminous giants in a number of clusters (e.g., Hut et al. 1992a, H92 hereafter; Fischer et al. (1993); Côté et al. (1994); Gebhardt et al. 1994; Mayor et al. 1995) find that a few percent of the stars show significant velocity variations when measured at $2-5$ epochs over time baselines of 2-10 years. Converting this "discovery frequency" into a true binary frequency is a difficult problem because surveys can typically only detect a subset, often a small subset, of all binaries. The simplest and probably best procedure is to report true binary frequencies for just the ranges of orbital period and mass ratio to which the survey is sensitive.

Figure 1 shows the fraction of binaries discovered as a function of period and mass ratio for a slightly updated version of the Pryor et al. (1989) and H92 sample of clusters, which consists of 1329 radial velocities for 392 stars in the globular clusters 47 Tuc, M2, M3, M12, M13, and M71. These curves are the result of Monte Carlo simulations identical to those described in Olszewski et al. (1996) (see also H92).

Longer-period binaries have low discovery probabilities because of their slowly-varying velocities and small velocity amplitudes. The discovery efficiency declines at short period because of the decreasing fraction of these 

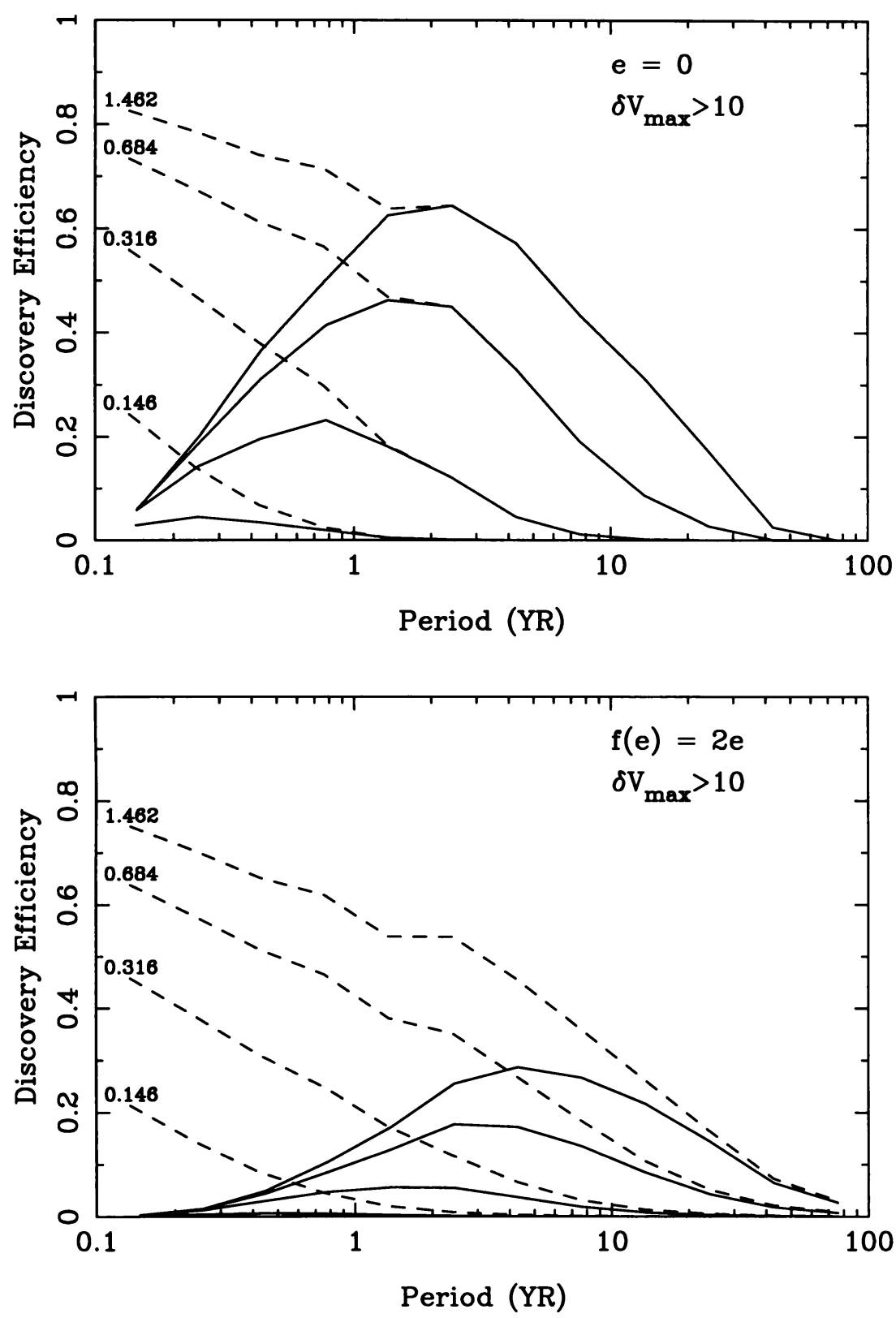

Figure 1. Binary discovery efficiency vs. orbital period for four bins in the mass ratio. The discovery criterion is a velocity range larger than $10 \mathrm{~km} \mathrm{~s}^{-1}$. The dashed lines show the effect of ignoring the size of the giants (i.e., these are the discovery efficiencies for main-sequence stars). Each of the four pairs of lines is for a different range of the binary mass ratio and is labeled with the mean value. The top panel assumes circular orbits, the bottom panel assumes a thermalized distribution of eccentricities. 
luminous giants whose radii are small enough to fit in the small orbital separations implied by short periods. The four pairs of curves in the figure are the discovery efficiency for four equal bins in the logarithm of the mass ratio in the range $0.33>\log (q)>-1.0$. Binaries with small mass ratios are difficult to detect because of the small velocity amplitude of the primary. The two panels of the figure show the significant uncertainty which the unknown eccentricity distribution implies for the discovery efficiency.

Figure 1 shows that this survey is most sensitive to binaries with orbital periods within a decade above and below the value of $2 \mathrm{yr}$. There are six stars with velocity ranges larger than $10 \mathrm{~km} \mathrm{~s}^{-1}$, yielding a discovery fraction of 0.015 with the $95 \%$ confidence interval $(0.0056,0.033)$ (see H92). The average binary discovery efficiency between a period of 0.2 and $20 \mathrm{yr}$ for the upper three mass-ratio bins of Fig. 1 is 0.29 for circular orbits and 0.10 for a thermalized distribution of eccentricities. These are slightly smaller than the values quoted in $\mathrm{H} 92$ because of small improvements in the simulations used to estimate the efficiencies. Correcting by these efficiencies yields binary frequencies of 0.027 per decade of period and 0.074 per decade for the two cases, respectively, for mass ratios larger than 0.22 . In other words, 0.027 or 0.074 of the stars in these clusters are the primaries of binaries with periods in an arbitrary decade in the vicinity of $2.0 \mathrm{yr}$ and with mass ratios larger than 0.22 .

Mass ratios larger than 1.0 are rare in the solar neighborhood (Duquennoy \& Mayor 1991, DM hereafter). In globular clusters, encounters between binaries and more massive single stars result in the heavier star being preferentially exchanged into the binary (Hills 1975; Sigurdsson \& Phinney 1993). Even so, more realistic efficiencies may result from averaging the values for the middle two mass-ratio bins in Fig. 1. This yields efficiencies of 0.20 and 0.068 and binary frequencies of 0.037 per decade $(e=0)$ and 0.11 per decade $(f(e)=2 e)$. The statistical uncertainties in all of these frequencies are about a factor of 2 at $95 \%$ confidence.

Nearby solar-type stars have a binary frequency of about 0.06 per decade in the vicinity of $2 \mathrm{yr}$ periods (DM). Thus, the average binary frequency in the above sample of globular clusters is between a factor of two lower and a factor of two higher than that in this field population, depending on the mass ratio and eccentricity distribution of the globular cluster binaries.

The DM sample has a roughly Gaussian period distribution extending from 1 day to $10^{7} \mathrm{yrs}$, with the most likely period being about $170 \mathrm{yrs}$. In globular clusters, however, binaries can be disrupted by encounters with single stars and binaries (Heggie 1975; Hills 1975) and, the longer the period, the larger the cross-section for gravitational encounters. The shortest period for which the binary can be disrupted by an encounter with a typical single star (i.e., binary binding energy equal to the typical kinetic energy 
of relative motion) is

$$
P=(65 \mathrm{yr})\left(\frac{5 \mathrm{~km} \mathrm{~s}^{-1}}{\sigma}\right)^{3} .
$$

Here $\sigma$ is the one-dimensional velocity dispersion of the cluster and all of the stars have been assumed to have a mass of $0.8 \mathcal{M}_{\odot}$. This period is also the approximate "watershed" longer than which encounters make the binary less bound on average and shorter than which they make the binary more bound (Hut 1983, see also the discussion in H92).

Stars with periods equal to or longer than the limit given by eq. (1) are on average disrupted in $1.5 \times 10^{10} \mathrm{yr}$ if $\sigma$ and the cluster number density of stars, $n$, satisfy

$$
\left(\frac{n}{1 \mathrm{pc}^{-3}}\right)\left(\frac{5 \mathrm{~km} \mathrm{~s}^{-1}}{\sigma}\right)^{3}>550 .
$$

This is based on the ionization rate of Hut \& Bahcall (1983) and again assumes $0.8 \mathcal{M}_{\odot}$ stars. This relation is satisfied at the centers of many globular clusters, though by widely varying margins. Equations (1) and (2) suggest that, even if globular clusters began with the same binary population as the DM sample, the overall binary frequency in globular clusters today would only be roughly half as large.

Binary stars with periods shorter than the boundary given by eq. (1) become more bound in encounters with single stars and this can eventually lead to their destruction through a stellar collision or to their ejection from the cluster by the recoil from an encounter. They can also be disrupted by encounters with other binaries. Particularly towards the centers of the denser clusters, binary stars of almost any period can have been destroyed by these processes. Mass segregation is expected to concentrate binaries towards the centers of clusters, so the observed numbers of binaries and their radial distribution will be the result of the interplay between segregation and destruction and this must be simulated in detail (Hut et al. 1992b; McMillan \& Hut 1994; Sigurdsson \& Phinney 1995).

Clearly it will be of interest to determine how the binary frequency varies between clusters with different densities, with radius in clusters, and with period. Evidence from radial velocity surveys on all of these points is still very preliminary. Pryor et al. (1991) report that the binary frequency seems somewhat higher in the low-density clusters NGC 5053 and NGC 5466. Yan (1995) is also surveying NGC 5053 and finding binaries, though no frequency has been reported yet. As summarized in $\mathrm{H} 92$, binary stars containing globular cluster giants have approximately the same radial 
distribution as the cluster light. Barden et al. (1995) report that velocity variables among stars near the base of the giant branch in M71 have the same radial distribution as the rest of the sample.

The Barden et al. (1995) sample and the Côté et al. (1995) sample of turnoff dwarfs in M4 are sensitive to shorter-period binaries than earlier studies (see also Yan 1995). They report preliminary binary frequencies of $0.05-0.08$ per decade and about 0.07 per decade, respectively. These are for $q>0.2$ and periods between a few days and a few years.

Studies with long time baselines can detect binaries with long periods. Côté et al. (1995) and Mayor et al. (1995) have recently reported studies for M22 and $\omega$ Cen, respectively, with time baselines of 10-20 yrs. Both studies find a binary frequency of 0.01-0.02 per decade for periods between a fraction of a year and 20-40 yr. These lower frequencies may be evidence for the destruction of longer-period binaries in globular clusters. However, note that the center of $\omega$ Cen does not satisfy eq. (2).

\section{Searches for Eclipsing Variables}

Mateo (1995) has recently reviewed this field and reports that there are now at least six globular clusters in which eclipsing variables are known and that this number is likely to increase rapidly. Accordingly, we only briefly discuss eclipsing binaries here (see also section 2.2 of H92; Shara, this volume; and Menzies, this volume).

Searches for eclipsing variables can only detect binary systems that are in contact or not far from it and thus have periods of at most a few days. The selection effects for detached systems favor the discovery of systems with separations of a few stellar radii and where both stars have similar luminosities. However, most of the systems discovered are contact mainsequence, or W UMa, systems. The strong distortion of the stellar shapes in these systems produce significant variations in the total luminosity even outside of the eclipses. Also, the thermal contact between the stars gives both stars equal surface brightnesses, making the detection of systems with large mass ratios quite feasible (see the discussion and references in Yan \& Mateo 1994 and H92).

Unfortunately, converting the number of W UMa systems detected into the number of progenitors is difficult. Main-sequence systems slowly spiral together due to gravitational radiation and, more importantly, magnetized winds (see the discussion and references in Eggen \& Iben 1989). Periods as long as 5 days might evolve into contact over the age of globular clusters. Even more uncertain is the lifetime of W UMa systems, with estimates ranging from $10^{8}$ to $10^{10}$ yrs (see Eggen \& Iben 1989). The number of W UMa systems seen is the product of the rate at which orbit (and stellar) 
evolution produces contact systems and the system lifetimes. Thus, problems remain with producing binary frequencies that can be compared to those from other techniques.

Currently, the only survey of eclipsing systems in a globular cluster that has yielded a binary frequency is the study of M71 by Yan \& Mateo (1994). They argue that all five eclipsing variables which they discovered are likely members of the cluster and calculate a binary frequency of about 0.013 for systems with any mass ratio and initial periods in the range 2.5-5 days, assuming a W UMa lifetime of $10^{9} \mathrm{yrs}$. The implied binary frequency per decade of period is 0.04 . This is about a factor of 2 larger than the DM frequency in this period range, but studies of more clusters and a better understanding of the evolution of W UMa systems are needed before it will be clear whether this is significant.

\section{Binary Sequences in the Color-Magnitude Diagram}

Unresolved main-sequence binaries produce "stars" in the cmd that are brighter and redder than the main sequence. Even if there is no peak in the distribution of binary mass ratios at $q=1$, there will be a peak in the number of binaries about $2.5 \log (2) \simeq 0.75 \mathrm{mag}$ above the main sequence (Romani \& Weinberg 1991, section 2.3 of H92). The reason is that, at a fixed color, the binaries will consist of one star that is brighter and one star that is fainter than a main sequence star with that color. To first order, the combined magnitude of the two stars does not change as the difference in brightness between the two stars increases. Uncertainties in measured magnitudes and colors do tend to wash out this peak (see figures 9 and 12 in H92), but a plateau of stars above the main-sequence remains.

Because of the measurement uncertainties, particular cmds will maximize the visibility of the binaries consisting of two main-sequence stars. The steeper the main-sequence in the cmd, the larger will be the vertical width of the sequence due to the smearing by uncertainties in the color direction. A steeper sequence also means that the color uncertainty, which is always larger than the magnitude uncertainty, contributes more to the intrinsic width of the sequence. Because binaries are always at most about $0.75 \mathrm{mag}$ brighter than the main-sequence, their visibility is greatest, for given measurement uncertainties, when the main-sequence has the shallowest slope in the cmd (e.g., Jaschek 1976). Thus, the redder color should always be used on the vertical axis of the cmd since this reduces the slope by a unit. Physically, this maximizes the contribution from the fainter and redder secondary.

These arguments also suggest that a long color baseline, such as $U-I$, would be good for detecting binaries in the cmd. The main-sequence colors 
from Edvardsson and Bell (1989) yield a slope in the $I$ vs. $U-I$ cmd of about 0.9 for the region from 1.0 to 3.0 magnitudes below the turnoff. The slopes for the $V$ vs. $B-V$ and $I$ vs. $V-I$ cmds are about 4.0. However, observations of globular cluster stars in the $U$ passband will tend to have larger uncertainties than those in redder bands, so quantitative estimates are necessary. The mass-magnitude relations also need to be taken into account since they determine the height above the main sequence of a binary with a specific mass ratio. Such quantitative comparisons of different cmds have not been carried out to our knowledge, but using $U$ magnitudes appears promising.

The largest problem with finding binaries in the cmd is the background of coalesced stellar images. This background can be evaluated using numerical experiments that add and recover artificial stars from the frames, but, if the crowding of images is significant, searching for binaries is very difficult (H92). The two globular clusters presenting the best cases for binary stars above the main sequence in ground-based data, E3 (McClure et al. 1985) and NGC 288 (Bolte 1992), have low central surface densities of stars, allowing data to be obtained close to the cluster centers. Thus, the advent of deep cmds for the centers of globular clusters based on highangular-resolution HST images will have a large impact on this field. The analysis of binary sequences in these cmds is still in a preliminary stage and thus we focus on the expected strength of "binary sequences" in the cmd and a few preliminary results.

The orbital period of the binaries plays no role in detecting them in the cmd. Thus, in globular clusters, systems with periods in the approximately 4 decades between about 5 days and 70 yrs are expected to be present and could be detected. The range of mass ratios detectable depends on the mass-luminosity relation along the main sequence. The steep relation below masses of about $0.15 \mathcal{M}_{\odot}$, visible for the first time in the deep cmd shown in Fig. 5 of Fahlman et al. (this volume), argues that binaries will be harder to detect fainter than about 5 mag below the turnoff. Brighter than that, however, the Alexander et al. (1995) $\mathrm{M}_{I}$-mass relations suggest that stars differing by a factor of two in mass will have $I$ magnitudes that differ by about 2.0. For a factor of three in mass, the difference is about 3.3 mag. Thus, binaries with $q=0.5$ and 0.33 will appear about 0.3 and $0.15 \mathrm{mag}$ above the main-sequence in the $(V, I) \mathrm{cmd}$, respectively. The likely detection threshold in $q$ is likely to be between these two values.

About 0.4 of the DM field binaries have $q>0.5$ and 0.6 have $q>0.33$. So roughly half of the binaries in a population with the DM $q$ distribution would be detectable in the cmd. If the binary frequency of 0.05 per decade of period with $q>0.2$ found from radial velocity studies extends over the whole 4 decade range of period and if the mass ratio distribution is similar 
to that from DM, then about 0.13 of the stars would be expected to appear in a "binary sequence" more than about 0.2 mag above the main sequence.

This estimate needs to be corrected downward slightly for systems with a white dwarf companion, which are detected by the radial velocity surveys and are not (at this location!) in the cmd. Ignoring mass transfer, assuming a power-law mass function with exponent $x(x=1.35$ being Salpeter $)$, and again adopting the DM mass ratio distribution suggests that between 0.15 $(x=2)$ and $0.30(x=1)$ of the binaries will have a dark companion. Thus, the fraction of stars expected in a binary sequence is around 0.10 .

Richer et al. (1995a) show the $U$ vs. $U-I$ cmd for stars at about 1 core radius $\left(1 r_{c}\right)$ in M4 based on photometry obtained with the HST. Richer et al. (1995b) report that approximately $4 \%$ of the stars in this diagram appear to be in a binary sequence. They also report two possible red dwarf - white dwarf binaries identified in the $U-V$ vs. $V-I$ diagram. Figure 5 in Fahlman et al. (this volume) shows the $V$ vs. $V-I$ cmd resulting from HST data for the $1 r_{c}$ M4 field and another field at $4 r_{c}$. The region of this cmd with $1.25<(V-I)_{0}<1.9$ has a main sequence that is free of bulge contamination and relatively shallow. In the $4 r_{c}$ field, the fraction of stars between 0.2 and $0.75 \mathrm{mag}$ above the main sequence is $2.5 \%$. Though the $V$ vs. $V-I \mathrm{cmd}$ is less powerful than the $I$ vs. $V-I$ diagram, these two very preliminary results suggest that the number of stars in binary sequences in M4 is smaller than the $10 \%$ predicted in the previous paragraph.

Perhaps we are seeing the effect of the destruction of binaries with periods longer than the few years that are best detected by radial velocity surveys. Bolte (1992) found that $10 \%$ of the stars in a field about 2.0 core radii from the center of NGC 288 were in a binary sequence with $q>0.7$. This cluster has a much lower central density than M4 and so it might be expected to have experienced less binary destruction. At least, it seems very likely that the distribution of binary periods in M4 is not as wide as in the solar neighborhood, in agreement with theoretical expectation. Much more work needs to be done to solidify these conclusions, however.

\section{Conclusion}

Binary stars clearly exist in globular clusters and there is tantalizing, but still very uncertain, evidence that the period distribution is different from that for binaries in the solar neighborhood. We are on the threshold of an exciting period where we will be able to use at least three different techniques to identify and study binaries: radial velocity variability, eclipses, and binary sequences in the cmd. These should be powerful tools for testing our theories for the dynamical evolution of globular clusters and their binary populations. 
Acknowledgments: The research of $\mathrm{CP}, \mathrm{RAB}, \mathrm{MB}$, and $\mathrm{HEB}$ on M4 is supported by NASA through grants from the Space Telescope Science Institute, which is operated by the Associated Universities for Research in Astronomy, Inc., under NASA conctract NAS5-26555. Support for the research of HBR, GGF, WEH, and DVB is provided by grants from the Natural Sciences and Engineering Research Council of Canada.

\section{References}

Alexander, D. R., Brocato, E., Cassisi, S., Castellani, V., Ciacio, F., \& Degl'Innocenti, S. (1996) submitted to $A \mathscr{B} A$

Barden, S. C., Armandroff, T. E., \& Pryor, C. (1995), in E. F. Milone (ed.), The Origins, Evolution and Destinies of Binaries In Clusters, in press

Bolte, M. (1992) ApJS, 82, 145

Côté, P., Fischer, P., Pryor, C., \& Welch, D. L. (1995), in E. F. Milone (ed.), The Origins, Evolution and Destinies of Binaries In Clusters, in press

Côté, P., Welch, D. L., Fischer, P., Da Costa, G. S., Tamblyn, P., Seitzer, P., \& Irwin, M. J. (1994), ApJS, 90, 83

Duquennoy, A., \& Mayor, M. (1991), $A \& A, \mathbf{2 4 8}, 485$

Edvardsson, B., \& Bell, R. A. (1989), MNRAS, 238, 1121

Eggen, O. J., \& Iben, I. (1989), $A J, 97,431$

Fischer, P., Welch, D. L., Mateo, M., \& Côté, P. (1993), AJ, 106, 1508

Gebhardt, K., Pryor, C., Williams, T. B., \& Hesser, J. E. (1994), AJ, 107, 2067

Heggie, D. C. (1975), MNRAS, 173, 729

Hills, J. G. (1975), $A J, \mathbf{8 0}, 809$

Hut, P. (1983), ApJ, 272, L29

Hut, P. \& Bahcall, J. (1983), ApJ, 268, 319

Hut, P., McMillan, S., Goodman, J., Mateo, M., Phinney, E.S., Pryor, C., Richer, H.B., Verbunt, F., \& Weinberg, M., (1992a), PASP, 104, 981

Hut, P., McMillan, S., \& Romani, R. (1992b), ApJ, 389, 527

Jaschek, C. (1976), $A \& A, \mathbf{5 0}, 185$

Mateo, M. (1995), in E. F. Milone (ed.), The Origins, Evolution and Destinies of Binaries In Clusters, in press

Mateo, M., Harris, H. C., Nemec, J. M., \& Olszewski, E. W. (1990), AJ, 100, 469

Mayor, M., Duquennoy, A., Udry, S., Andersen, J., \& Nordström, B. (1995), in E. F. Milone (ed.), The Origins, Evolution and Destinies of Binaries In Clusters, in press

McClure, R. D., Hesser, J. E., Stetson, P. B., \& Stryker, L. L. (1985), PASP, 97, 605

McMillan, S. \& Hut, P. (1994), ApJ, 427, 793

Olszewski, E. W., Pryor, C., \& Armandroff, T. E. (1996), AJ, in press, Feb.

Pryor, C., McClure, R.D., Hesser, J.E., \& Fletcher, J.M. (1989), in D. Merritt (ed.), Dynamics of Dense Stellar Systems, Cambridge University Press, Cambridge, p. 175

Pryor, C., Schommer, R. A., \& Olszewski, E. W. (1991), in K. Janes (ed.), The Formation and Evolution of Star Clusters, ASP, San Francisco, p. 439

Richer, H.B. et al. (1995a), ApJL, 451, L17

Richer, H. B. et al. (1995b), in E. F. Milone (ed.), The Origins, Evolution and Destinies of Binaries In Clusters, in press

Romani, R. W., \& Weinberg, M. D. (1991), ApJ, 372, 487

Sigurdsson, S., \& Phinney, E. S. (1993), ApJ, 415, 631

Sigurdsson, S., \& Phinney, E. S. (1995), ApJS, 99, 609

Yan, L., \& Mateo, M. (1994), $A J, 108,1810$

Yan, L. (1995), Caltech Ph.D. thesis 